PROCEEDINGS OF THE

AMERICAN MATHEMATICAL SOCIETY

Volume 132, Number 8, Pages 2357-2360

S 0002-9939(04)07468-4

Article electronically published on March 25, 2004

\title{
THE EQUATIONS DETERMINING INTERMEDIATE INTEGRALS FOR MONGE-AMPÈRE PDE
}

\author{
R. J. ALONSO-BLANCO
}

(Communicated by David S. Tartakoff)

\begin{abstract}
In this note we will find the differential equations determining the intermediate integrals for Monge-Ampère equations in an arbitrary number of variables.
\end{abstract}

An intermediate integral of a PDE system $\mathcal{E}$ is another PDE system of lower order, whose solutions are also solutions of $\mathcal{E}$. The Goursat method for integrating classical Monge-Ampère (second-order) equations is based on the search of intermediate integrals 2]. In this paper we will obtain a computable criterion which determines when a first-order PDE is an intermediate integral of a (generalized) Monge-Ampère equation in an arbitrary number of variables. The derivation of this criterion relies only on well-known properties of first-order PDE systems and some elementary linear algebra.

\section{Preliminaries: First-order PDE in a single unknown}

In this section we will recall a few notions about first-order PDEs. For further information refer to $[3]$ and $[5]$.

Let $M$ be an $n$-dimensional manifold and $J^{1} M$ the bundle of 1 -jets of functions $M \rightarrow \mathbb{R} . J^{1} M$ is a contact manifold endowed with the canonical 1-form $\theta$ which in appropriate local coordinates $\left\{x_{i}, z, p_{i}\right\}, 1 \leq i \leq n$, has the expression $\theta=$ $d z-\sum_{i=1}^{n} p_{i} d x_{i}$.

Definition 1.1. A first-order $P D E$ in a single unknown in $n$ variables is given by means of a hypersurface $\mathcal{E}_{F}=\{F=0\} \subset J^{1} M, F \in \mathcal{C}^{\infty}\left(J^{1} M\right)$. A (generalized) solution of the equation $\mathcal{E}_{F}$ is an $n$-dimensional solution of the exterior differential system generated by $F$ and $\theta$.

From now on, we will take a fixed function $F \in \mathcal{C}^{\infty}\left(J^{1} M\right)$, and we will consider the family of equations $\mathcal{E}_{F+\text { const }}$ (one for each chosen constant).

Definition 1.2. A point $e \in J^{1} M$ is called non-singular for $F$ if $d_{e} F$ and $\theta_{e}$ are linearly independent. Let us denote the set of non-singular points of $F$ by $U_{F}$. A solution $Z$ of $\mathcal{E}_{F+\text { const }}$ is said to be non-singular if $Z \subset U_{F}$.

Received by the editors May 5, 2003.

2000 Mathematics Subject Classification. Primary 35A30, 58A15.

Key words and phrases. Nonlinear partial differential equation, Monge-Ampère equation, jet, intermediate integral, contact manifold.

The author was partially funded by Junta de Castilla y León under contract SA077/03.

(C)2004 American Mathematical Society 
In the following two lemmas we will recall some well-known facts which will be needed in the next section.

Lemma 1.3. Let $e \in U_{F}$, and let us denote by $C_{F, e}$ the linear span of the tangent spaces $T_{e} Z$, where $Z$ is a solution of $\mathcal{E}_{F-F(e)}$. Then,

$$
C_{F, e}=\left\{D \in T_{e} J^{1} M \mid i_{D} \theta_{e}=i_{D} d_{e} F=0\right\} .
$$

Lemma 1.4. There is a unique contact tangent field $\bar{X}_{F}$ such that $i_{\bar{X}_{F}} \theta=F$. In addition, $X_{F}=F \bar{X}_{1}-\bar{X}_{F}$ generates the characteristic system of the Pfaff system $\{\theta, d F\}$ and also

(1) $X_{F} F=i_{X_{F}} d F=0$,

(2) $i_{X_{F}} \theta=0$,

(3) $\left(X_{F}\right)_{e} \neq 0$ for every $e \in U_{F}$,

(4) $X_{F}$ is tangent to any solution of each equation $\mathcal{E}_{F+\text { const }}$.

\section{Intermediate Integrals FOR Monge-Ampère EQUATIONS}

In this section we will recall the definition of Monge-Ampère equations and prove our main result.

Definition 2.1. Let $\omega$ be a differential $n$-form on $J^{1} M$. The Monge-Ampère equation defined by $\omega$ is given by the exterior differential system

$$
\mathcal{E}_{\omega} \stackrel{\text { def }}{=}\{\theta, \omega\} .
$$

Thus, an $n$-dimensional submanifold $Z \subset J^{1} M$ is a solution of $\mathcal{E}_{\omega}$ if

$$
\left.\omega\right|_{Z}=0,\left.\quad \theta\right|_{Z}=0 .
$$

Definition 2.2. A function $F \in \mathcal{C}^{\infty}\left(J^{1} M\right)$ is said to be an intermediate integral of $\mathcal{E}_{\omega}$ if the non-singular solutions of $\mathcal{E}_{F+\text { const }}$ are also solutions of $\mathcal{E}_{\omega}$ for every constant const.

Let $\omega$ be a differential $n$-form on $J^{1} M$ and $F \in \mathcal{C}^{\infty}\left(J^{1} M\right)$. On a neighborhood of each point in $U_{F}$ we choose a coframe $\left\{\theta, d F, \sigma_{1}, \ldots, \sigma_{2 n-1}\right\}$. This way we can write

$$
\omega=\theta \wedge \lambda+d F \wedge \mu+\omega^{\prime}
$$

where $\omega^{\prime}$ does not contain $\theta$ or $d F$. Moreover, $\left.\omega\right|_{Z}=\left.\omega^{\prime}\right|_{Z}$ when $Z$ is a solution of $\mathcal{E}_{F+\text { const }}$.

Theorem 2.3. A function $F \in \mathcal{C}^{\infty}\left(J^{1} M\right)$ is an intermediate integral of $\mathcal{E}_{\omega}$ if and only if

$$
\theta \wedge d F \wedge i_{X_{F}} \omega=0
$$

on the open set $U_{F}$.

Proof. Without loss of generality, we can work over an open subset $V \subset U_{F}$ where the above coframe was defined.

From (21) and items (1)-(2) of Lemma 1.4 we derive that (3) is equivalent to

$$
i_{X_{F}} \omega^{\prime}=0 .
$$

Let us assume that (3) (or (44) holds. If $Z$ is a solution of $\mathcal{E}_{F+\text { const }}$ we can restrict $X_{F}$ to $Z$ (item (4) of Lemma 1.4). Then

$$
\left.i_{\left.X_{F}\right|_{Z}} \omega^{\prime}\right|_{Z}=\left.\left(i_{X_{F}} \omega^{\prime}\right)\right|_{Z}=0 .
$$


On the other hand, the inner contraction of a non-vanishing $n$-form by a nonvanishing tangent field (item (3) of Lemma 1.4) on an $n$-dimensional manifold cannot be zero at any point. As a result we obtain $\left.\omega^{\prime}\right|_{Z}=0$ and therefore, $\left.\omega\right|_{Z}=0$. In other words, $F$ is an intermediate integral of $\mathcal{E}_{\omega}$.

Conversely, let $F$ be an intermediate integral of $\mathcal{E}_{\omega}$. This way

$$
\left.\left(i_{X_{F}} \omega^{\prime}\right)\right|_{Z}=\left.i_{X_{F} \mid Z} \omega^{\prime}\right|_{Z}=\left.i_{X_{F} \mid Z} \omega\right|_{Z}=0
$$

for every solution $Z$ of $\mathcal{E}_{F+\text { const }}$.

By the definition of $C_{F, e}$, we can also obtain $\left.\left(i_{X_{F}} \omega^{\prime}\right)\right|_{C_{F, e}}=0$, for each point $e \in V$. In addition, $C_{F, e}$ is the incident subspace to $\theta_{e}$ and $d_{e} F$ (Lemma 1.3). Recall, also, that $\omega_{e}^{\prime}$ does not contain $\theta_{e}$ or $d_{e} F$ (in the coframe we are using). In conclusion, $\left(i_{X_{F}} \omega^{\prime}\right)_{e}=0$ for every $e$. This way, $i_{X_{F}} \omega^{\prime}=0$ and thus (3) holds.

Remarks 2.4. 1) Condition (3) is a first-order PDE for the unknown function $F$. As a consequence, we can find solutions of $\mathcal{E}_{\omega}$ by solving first-order equations: equation (3) and $\mathcal{E}_{F+\text { const }}$.

2) Any contact manifold is locally equivalent to $J^{1} M$ for an appropriate $M$. Thus, all the results in this paper are locally valid for arbitrary contact manifolds. As a matter of fact, if a global contact structure 1-form $\theta$ exists, the field $X_{F}$ can be defined and the result can be directly translated to this case.

3) A criterion was also given by Zil'bergleit [6] who uses the more sophisticated framework of Lychagin [4] on contact geometry: effective forms, $\mathfrak{s l}(2)$ representations, Hodge-Lepage expansions, operators $T$ and $\perp$, etc. In addition, the criterion in 6], unlike Theorem 2.3, must be applied only on "effective" forms $\omega$.

4) We can define an intermediate integral of $\mathcal{E}_{\omega}$ in a more general sense, that is, as a function $F$ such that all the solutions of $\mathcal{E}_{F}$ are also solutions of $\mathcal{E}_{\omega}$ (i.e., just const $=0$ is considered in Definition 2.2. . Then, Theorem 2.3 should be modified by requiring $\theta \wedge d F \wedge i_{X_{F}} \omega=0$ only at the points of the hypersurface $\{F=0\} \subset J^{1} M$.

5) Let us consider the case $\operatorname{dim} M=2, \omega=\omega^{1} \wedge \omega^{2}$. If $d F$ belongs to the characteristic system $\left\{\theta, \omega^{1}, \omega^{2}\right\}$, then $\theta \wedge d F \wedge \omega=0$. By applying Theorem 2.3 we can see that $F$ is an intermediate integral of $\mathcal{E}_{\omega}$. This way, we recover the criterion given for example in [1] (where a detailed analysis on the possibility of singular solutions was done). However, this result states just a sufficient condition. For example, it is not difficult to check that $F=p_{1}+x_{2}$ is an intermediate integral for the equation

$$
\frac{\partial^{2} z}{\partial x_{1}^{2}} \frac{\partial^{2} z}{\partial x_{2}^{2}}-\left(\frac{\partial^{2} z}{\partial x_{1} \partial x_{2}}\right)^{2}+1=0,
$$

which is obtained from $\omega=\omega^{1} \wedge \omega^{2}, \omega^{1}=d p_{2}+d x_{1}$, and $\omega^{2}=-d p_{1}+d x_{2}$. Nevertheless, $d F \notin\left\{\theta, \omega^{1}, \omega^{2}\right\}$ (on the other hand, $i_{X_{F}} \omega=0$ and definitely Theorem 2.3 can be applied).

\section{ACKNOWLEDGMENTS}

I would like to express my gratitude to Prof. J. Muñoz for his interest and his help in my work. Also, thanks to S. Jiménez and J. Rodríguez for useful discussions and suggestions. 


\section{REFERENCES}

1. J. N. Clelland, On the intermediate integral for Monge-Ampère equations, Proc. Amer. Math. Soc. 128 (2000), no. 2, 527-531. MR 2000c:35046

2. E. Goursat, Leçons sur l'intégration des équations aux dérivées partielles du second ordre, vol. I, Gauthier-Villars, Paris, 1890.

3. V. V. Lychagin, Local classification of nonlinear first order partial differential equations, Uspekhi Math. Nauk. 30 (Russian); English translation, Russian Math. Surveys 30 (1975), $105-176$.

4. V. V. Lychagin, Contact geometry and second-order nonlinear differential equations, Uspekhi Mat. Nauk 34:1 (1979), 137-165 (Russian); English translation, Russian Math. Surveys 34:1 (1979), 149-180. MR 80h:58057

5. J. Muñoz-Díaz, Ecuaciones diferenciales I, Universidad de Salamanca, Salamanca, 1982. (Spanish)

6. L. V. Zil'bergleìt, Intermediate integrals of the Monge-Ampère equations. (Russian) Izv. Vyssh. Uchebn. Zaved. Mat. 1999, , no. 9, 16-25; translation in Russian Math. (Iz. VUZ) 43 (1999), no. 9, 13-22 (2000). MR 2001f:58008

Departamento de Matemáticas, Universidad de Salamanca, Plaza de la Merced 1-4, E-37008 Salamanca, Spain

E-mail address: ricardo@usal.es 